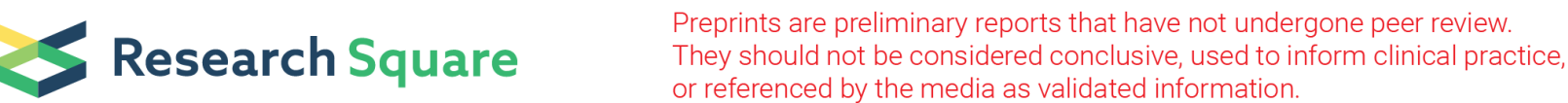

\section{Incidence and Predictors of Sever Adverse Drug Reactions Among Patients on Antiretroviral Therapy at Debre Markos Referral Hospital, Northwest Ethiopia}

Getiye Dejenu Kibret ( $\nabla$ dgetiye@gmail.com )

Debre Markos University College of Health Science https://orcid.org/0000-0002-1374-5744

Tadesse Awoke Ayele

University of Gondar

Adino Tesfahun

University of Gondar

Research article

Keywords: Adverse Drug Reactions, Antiretroviral, HIV, Drug toxicity

Posted Date: April 29th, 2019

DOl: https://doi.org/10.21203/rs.2.9264/v2

License: (c) (1) This work is licensed under a Creative Commons Attribution 4.0 International License.

Read Full License 


\section{Abstract}

Background Due to its nature of chronicity and the trend of using more drugs for patients with HIV, antiretroviral toxicity becomes a major challenge of HIV management. Evidences revealed that magnitude of experiencing at least one form of drug toxicity in different setting have been reached up to $90 \%$ among patients on antiretroviral therapy. Objective The main aim of this study was to estimate the incidence and predictors of severe adverse drug reactions among People Living with Human Immunodeficiency Virus (PLHIV) at Debre Markos Referral Hospital. Methods Retrospective follow up study with survival analysis was carried out among people living with Human Immunodeficiency virus. Mean survival time of the cohort was estimated using Kaplan-Meier method. To model the relationship between explanatory variables and the time to adverse drug reaction, Cox-proportional hazard regression model was fitted. Results Out of the total 485 participants 67 (13.81\%; 95\% C.I: 10.7\%, 16.8\%) had experienced sever adverse drug reactions (ADRs). The incidence rate of severe adverse drug reaction development was 3 per 100-person years. Patients residing out of the catchment area to the facility were $73 \%$ at higher risk to develop ADR at any time, compared to those living within the catchment area (AHR=1.73; 95\% C.I: 1.04, 2.86). The risk of ADRs among patients with baseline WHO clinical stage of III and IV was 2.59 times higher at any time compared to those with WHO stages I and II (95\% C.I: 1.54, 4.36). Conclusion The incidence of adverse drug reactions was relatively lower than reported in different parts of Ethiopia and other African countries. However, the overall burden in the 10 years period was still high. Health professionals working in the ART clinic need to give special attention for patients coming from outside of catchment areas, commercial sex workers and drivers and patients on advanced WHO clinical stages to prevent ADR development among these groups.

\section{Background}

HIV infected more than 76.1 million people and claimed 35.0 million lives so far since its epidemic has underway. By the end of 2016, about 36.7 million people were living with HIV. Ninety four percent (34.5 million) were adult population and 17.7 million were women $15+$ years. In the same year, 1.8 million people become newly infected [1]. Sub-Saharan Africa carries the highest burden of HIV and AIDS, with an estimated proportion of $71 \%$ the global total [2]. East and Southern Africa region is the most affected segment with HIV pandemic [3].

Global scale-up of antiretroviral therapy reaching its coverage to $46 \%$ at the end of 2015 [4] has been the primary contributor to a $48 \%$ decline in deaths from AIDS-related causes, from a peak of 1.9 million in 2005 to 1.0 in 2016 [3]. However, still there are a lot of challenges hampering the progress, of which drug toxicity is the main one.

Adverse drug reactions (ADRs), here after drug toxicity or side effects (interchangeably for this study context), are defined as "an appreciably harmful or unpleasant reaction, resulting from an intervention related to the use of a medicinal product, which predicts hazard from future administration and warrants prevention or specific treatment, or alteration of the dosage regimen, or withdrawal of the product [5]." 
Serious reactions include those result in death, are life-threatening, result in hospitalization or prolongation of hospitalization, those result in regimen change or discontinuation and those result in permanent harm or disability [6].

Due to its nature of chronicity and the trend of using more drugs for patients with HIV, antiretroviral toxicity becomes a major challenge of HIV management. Evidences revealed that magnitude of experiencing at least one form of drug toxicity in different setting have been reached up to $90 \%$ of PLHIV. Higher incidence rate of 52/100 PY was also reported from India [7].

The UNAIDS $90-90-90$ targets propose that to end the HIV epidemic by $2030,90 \%$ of persons living with HIV worldwide should be diagnosed, $90 \%$ of diagnosed PLWH should be on antiretroviral therapy and $90 \%$ of PLWH on ART should have suppressed viral load by 2020 [8].

Another good pace is, what has been recommended in the $2016 \mathrm{WHO}$ consolidated guidelines on the use of ARV drugs for treating and preventing HIV infection. WHO launched the test and treat (Treat All) policy for all member Nations [6]. As a result, more people will start ART earlier and remain on treatment for a longer period on a wider scale. Consequently, it is not tough to assume how the load of drug toxicity will intensify. That's why WHO recommended for integrating ARV toxicity surveillance into HIV monitoring and evaluation programs [9].

Recommendations for optimizing ARV toxicity surveillance approaches include "nesting ARV cohort event monitoring in a few centers and strengthening surveillance of hospitalizations due to ARV toxicity at selected hospitals" [10].

Adverse drug reaction is also an obstacle for ART treatment adherence. Evidences indicated that presence of adverse drug reaction adversely affects ART drug compliance. A prospective study in Ethiopia, found that more than $85.7 \%$ of the cohort experienced at least one ADR, and almost a third experienced a severe ADR in the first 12 weeks of therapy. Most ADRs were developed within the first four weeks of treatment initiation. Incidence rates for any ADR and severe ADRs were 14.8 and 3.2 per 100 person-months follow ups respectively. In relation to these loads, they found out that patients with severe ADRs were less likely to adhere [11].

In realizing the 90-90-90 ambitious goals, working against treatment failure and ART drug adherence are important pillars. In this regard, drug toxicity and side effects are the main challenges exhausting follow up, hampering adherence and thereby, leading to poor treatment outcomes. ART drug toxicity related distress has been evidenced as one of the detrimental factor against treatment adherence [11]. Especially, achieving the third 90 will be fantasy unless robust efforts are executed.

In the context of "Treat All" approach, drug toxicity will be more tough for low income countries like Ethiopia, where anew recommended treatments with lower risk of ADRs are not early updated. Developing contextually feasible effective ARV toxicity monitoring systems is a complex task and effort demanding. The WHO technical review meeting on antiretroviral drug toxicity surveillance in 2013 recommended a 
focus on operational research in order to optimize implementation modalities [9]. Several previous studies regarding adverse drug reactions of ART estimate incidences of all forms of ADRs and did not focused on the sever form, which is the main determinant for adherence. Therefore, this study aimed to estimate the incidence of sever adverse drug reactions and its predictors among patients on antiretroviral therapy.

\section{Methods}

\section{Study Area and Period}

The study was conducted at Debre Markos Referral Hospital, Amhara Regional State. Debre Markos Referral Hospital is found in Debre markos town, the main city of East Gojjam Administrative Zone. It is located $300 \mathrm{~km}$ away from Addis Ababa, the capital city of Ethiopia and $264 \mathrm{~km}$ from Bahir Dar capital city of Amhara Regional State. In the town there are four health centers, more than eight private clinics and one Referral Hospital delivering health care. Debre Markos Referral Hospital serve for more than 3.5 million people in its catchment area. It provides comprehensive HIV/AIDS care and support services for more than 3716 people on follow up since 2005. Debre Markos town is seventh ranked by the load of total population with known HIV status cases as of April 2017 national report [12]. The study used secondary data of patients enrolled from January 2008 to January 2018. The data were retrieved from March to the end of May, 2018.

\section{Study Design and population}

Retrospective follow up study design with survival data analysis was carried out. The target population for this study were all adults who have been on ART follow up since January 2008 to January 2018 at Debre Markos Referral Hospital. Those who had at least one repeated follow up visit were included and patients with incomplete important baseline registries (WHO clinical stage, functional status, regimen type) were excluded from the analysis.

\section{Sampling Technique and Sample Size Determination}

The list of all eligible participants on ART follow up was obtained in soft copy from the ART clinic. Computer generated simple random sampling method using medical registration numbers was carried out to select patient records.

Sample size was calculated in STATA 14.1 based on sample size determination formula for survival analysis, considering $10 \%$ contingency, the final sample size analyzed was 485 .

\section{Study Variables}

The primary outcome of interest for this study is time to the development of severe adverse drug reactions. The explanatory variables include the socio-demography factors, baseline characteristics and follow up measurements. 
Severe adverse drug reaction: In this study severe ADR is defined as having any one of the following features recorded as drug reaction complaints for seeking care and resulted in either of regimen change, discontinuation and /or in-patient care (including all available laboratory test results): diarrhea, Hepatotoxicity, Peripheral neuropathy, Severe skin rash and hypersensitivity reaction (Stevens-Johnson syndrome), anemia, Pancreatitis, abdominal pain, jaundice, fat changes, anxiety, depression, vomiting and other rare conditions.

Generally grade 3 and 4 of ARV toxicity according to WHO clinical grading were considered as severe adverse drug reactions as follows:

Severer nausea: Severe discomfort or minimal intake for $>3$ days

Severer vomiting: Severe vomiting of all foods/fluids in 24 hours, orthostatic hypotension, or necessity for IV therapy

Severer diarrhea: severe diarrhea, with orthostatic hypotension, or necessity for IV therapy Severer headache: Severe or requires narcotic therapy

Severer allergic reaction: Angioedema or anaphylaxis

Severer fatigue: Normal activity reduced $>50 \%$

Event: Severe adverse drug reaction

Censored: loss to follow up, transfer out, not diagnosed with drug toxicity until the end of the follow up Survival time: Time from ART initiation to every ADR occurrence

Catchment area: Catchment area for ART follow up is defined as the most nearby health facility for patients which can provide the follow up services including counseling and treatment refilling. In this study context, patients coming from outside of Debre Markos referral hospital for ART follow up, bypassing another nearby health facility were considered as coming from out of catchment area.

\section{Data Collection Procedure and Quality Control}

Structured data extraction check list developed based on the ART receiving and follow up guide line was used. Trained health professionals working in the ART clinic were recruited for data collection and supervision.

Pretest was done to check for tool consistency and relevance. Two days training was given for data collectors and supervisors. Strict follow up and supervision was carried out during data collection time.

\section{Data processing and analysis}

Data were entered using Epi Data version 4.2 and analyzed using R programing statistical software. Kaplan-Meier (KM) survival curve with Log- rank test was used to compare survival curves for categorical 
explanatory variables. Median and mean survival times were estimated using KM method. To model the relationship between explanatory variables and the outcome variable, Cox-proportional hazard regression model was implemented. For the evaluation of proportional hazards assumption, log-log survival curves, Schoenfeld goodness of fit test and time-dependent covariates approach were used. Goodness of fit of the final model was checked using Cox-Snell residuals with the Nelson-Aalen cumulative hazard function graph.

\section{Results}

\section{Baseline and Sociodemographic Characteristics}

In this retrospective follow up study, a total of 485 patient cards were retrieved and analyzed. The median age of participants was 33 years (Q1, Q3: 27,40). Two hundred seventy-three $(44.7 \%)$ of the study participants were outside of the catchment area and $59.8 \%$ were females (Table 1).

Table 1: Baseline and Socio-demographic characteristics of patients on ART, Debre Markos Referral Hospital, 2008 -2018. 


\begin{tabular}{|c|c|c|c|}
\hline \multicolumn{2}{|l|}{ Characteristics } & \multirow{2}{*}{$\begin{array}{l}\text { Frequency } \\
63\end{array}$} & \multirow{2}{*}{$\begin{array}{l}\text { Percentage } \\
13.0\end{array}$} \\
\hline Age in years & $15-24$ & & \\
\hline & $25-34$ & 203 & 41.9 \\
\hline & $35-44$ & 146 & 30.1 \\
\hline & $>=45$ & 73 & 15.1 \\
\hline \multirow[t]{4}{*}{ Marital status } & Not married & 82 & 16.9 \\
\hline & Married & 224 & 46.2 \\
\hline & Divorced & 117 & 24.1 \\
\hline & Widowed & 62 & 12.8 \\
\hline \multirow[t]{6}{*}{ Occupation } & Sex workers & 15 & 3.1 \\
\hline & Drivers & 22 & 4.5 \\
\hline & Contract employee(private) & 312 & 64.3 \\
\hline & Farmer & 52 & 10.7 \\
\hline & Government employee & 79 & 16.3 \\
\hline & Others* & 5 & 1.0 \\
\hline \multirow[t]{3}{*}{ Religion } & Orthodox & 432 & 89.1 \\
\hline & Muslim & 45 & 9.3 \\
\hline & Other** & 8 & 1.6 \\
\hline \multirow[t]{3}{*}{ Education } & No formal education & 151 & 31.1 \\
\hline & Primary education & 132 & 27.2 \\
\hline & Secondary education & 202 & 41.6 \\
\hline \multirow[t]{3}{*}{ BMI } & Under weight & 167 & 34.4 \\
\hline & Normal & 293 & 60.4 \\
\hline & Overweight and obese & 25 & 5.2 \\
\hline \multirow[t]{2}{*}{ CD4 count } & $\mathrm{CD} 4<=200$ & 291 & 60.0 \\
\hline & CD4 >200 & 194 & 40.0 \\
\hline \multirow[t]{3}{*}{ Baseline regimen } & $1 \mathrm{a}=\mathrm{d} 4 \mathrm{t}-3 \mathrm{TC}-\mathrm{NVP}$ & 76 & 15.7 \\
\hline & $1 b=d 4 t-3 T C-E F V$ & 68 & 14.0 \\
\hline & 1c=AZT-3TC-NVP & 76 & 15.7 \\
\hline
\end{tabular}




\begin{tabular}{lll}
$1 \mathrm{~d}=$ =AZT-3TC-EFV & 42 & 8.7 \\
\hline $1 \mathrm{e}=$ TDF-3TC-EFV & 208 & 42.9 \\
$1 \mathrm{f}=$ =TDF+3TC+NVP & 7 & 1.4 \\
\hline $1 \mathrm{~g}=$ ABC+3TC+EFV & 7 & 1.4 \\
\hline 1h=ABC+3TC+NVP & 1 & 0.2
\end{tabular}

*Students, unemployed ** protestant, catholic

Incidence Rates of Adverse Drug Reactions

Out of the total 485 participants 67 (13.81\%; 95\% C.I: 10.7\%, 16.8\%) had experienced sever adverse drug reactions (ADRs).

Of the total 67 observed events anemia account for the majority $28(41.79 \%)$ of ADRs, followed by peripheral neuropathy $19(28.36 \%)$ and serious skin reactions $9(13.43 \%)$. The remaining forms of ADRs were lipodystrophy $4(5.97 \%)$, vomiting $4(5.97 \%)$, renal failure $2(2.98 \%)$ and hepatotoxicity $1(1.49 \%)$. The incidence rate of ADR development was 3 (95\% C.I: 2.4, 3.86) per 100-person years, with a total of 2202.7 follow up years. The incidence rates in male and female were 19.3 and 38.5 per 1000-person years of follow up respectively. In the other hand, the incidence rate of ADR was lower among those who took cotrimoxazole preventive therapy compared to their counterparts (27.8/1000 PY and 40.5/1000PY) respectively. The incidence rate varies at different interval of the cohort (Table 2)

The overall cohort, censoring and event median follow up times were 51 (Q1, Q3: 17, 90), 56 (Q1, Q3: $14.75,96.25)$ and $47(\mathrm{Q} 1, \mathrm{Q} 3: 31,56)$ months respectively. Due to the smaller proportion of the event in the cohort, the median survival time was not estimable. So, we used the survival mean to estimate the mean survival time. In this regard, the survival mean will be estimated better considering the maximum event time, which is reported as restricted mean survival time [13]. The estimated mean survival time using the restricted mean was 73.72 (95\% C.I: 71.84, 75.60) months. In the other hand, the estimated median survival time among those experienced the event of interest (ADR) was 47 (95\% C.I: 40.8, 53) months.

Majority of the ADRs were encountered among those whose baseline ART regimen was d4T-3TC-NVP (43.3\%) followed by d4T-3TC-EFV (37.3\%). The proportion was also high among those with baseline WHO stage of III and IV (28.4\% and $43.3 \%)$ respectively.

Table 2: Person time follow up and incidence rates of ADR among patients on ART, Debre Markos Referral Hospital 2008 to 2018. 


\begin{tabular}{llllll} 
Cohort (years) & person-time & failures & rate & $95 \%$ Conf. & Interval \\
\hline$(0-1]$ & 435.25 & 10 & 0.02 & 0.012 & 0.042 \\
\hline$(1-2]$ & 364.42 & 4 & 0.01 & 0.004 & 0.029 \\
\hline$(2-3]$ & 329.25 & 10 & 0.03 & 0.016 & 0.056 \\
\hline$(3-4]$ & 281.58 & 14 & 0.05 & 0.029 & 0.083 \\
\hline$(4-5]$ & 230.92 & 24 & 0.10 & 0.069 & 0.155 \\
$>5$ & 561.25 & 5 & 0.01 & 0.004 & 0.021
\end{tabular}

\section{Survival probability}

Majority (92.5\%) of the ADRs occurred up to the end of 5 th year of follow up, with more than half the events encountered in the interval between three and five years (Table 3 ).

Table 3: Cumulative survival probability at different time intervals among patients on ART, Debre Markos Referral Hospital, 2008 -2018.

\begin{tabular}{lllll} 
Time (year) & Beginning Total & Fail & Cumulative Survival & $95 \%$ C. I \\
\hline 1 & 393 & 10 & 0.98 & $(0.96,0.99)$ \\
\hline 3 & 306 & 14 & 0.94 & $(0.91,0.96)$ \\
\hline 5 & 201 & 38 & 0.80 & $(0.75,0.84)$ \\
\hline 7 & 145 & 5 & 0.78 & $(0.73,0.82)$ \\
\hline 10 & 6 & 0 & 0.78 & $(0.73,0.82)$ \\
\hline
\end{tabular}

Figure 1: Cumulative survival Kaplan Meier curve for time to the development of ADRs among HIV patients on ART, Debre Markos Referral Hospital.

\section{Factors Associated with Time to the Development of ADRs in Bivariable Analysis}

Variables with p-values of less than 0.25 from univariable analysis were screened for multivariable analysis in Cox proportional hazard model. Variables including gender, residence, baseline WHO stage, occupation, baseline regimen, regimen change, baseline CD4 cell count, taking cotrimoxazole preventive therapy, baseline BMI, experience of TB infection and baseline functional status candidate for multivariable analysis. Some of the variables also were significant at $p-$ value of 0.05 .

Female were less survivors to develop ADR than males (Figure 3)

Figure 2: Kaplan Meier curves for time to the development of ADRs among HIV patients on ART by gender, Debre Markos Referral Hospital 2008 to 2018. 
Those from outside of the catchment area to Debre Markos referral hospital were at lower survival compared to those living within the catchment area.

Figure 3: Kaplan Meier curves for time to the development of ADRs among HIV patients on ART by source of residence, Debre Markos Referral Hospital 2008 to 2018.

\section{Goodness of Fit of the Final Model}

The goodness of fit of the final Cox regression model was evaluated using the estimate of Cox-Snell residuals drawn against the Nelson-Aalen cumulative hazard function (Figure 5).

Figure 4:Goodness of fit of the final Cox proportional hazards regression model using Cox Snell Residuals

The hazard function follows the forty-five-degree line very closely except for large values of time. It is very common for models with censored data to have some wiggling at large values of time and it is not something which should cause much concern [14]. Therefore, we would conclude that the final model fits the data well.

\section{Predictors of Time to Development of Severe ADRS among Patients on ART}

After checking for the Cox proportional hazard assumptions using the graphical, statistical and time dependent methods, the multivariable Cox proportional model was run.

Those with livelihood of commercial sex work and car driving were 2.78 times at higher risk of developing ADR compared to those with contract employment (95\% C.I: 1.31, 5.92). Patients residing out of the catchment area to the facility were $73 \%$ at higher risk to develop ADR at any time, compared to those living within the catchment area (AHR=1.73; 95\% C.I: 1.04, 2.86). The risk of ADRs among patients with baseline WHO clinical stage of III and IV was 2.59 times higher at any time compared to those with WHO stages I and II (95\% C.I: $1.54,4.36)$.

Patients who ever took anti-TB prophylaxis were 2.83 times more likely to develop adverse drug reactions at any time in the follow up compared to those with no such experience (95\% C.I: 1.61, 4.96). In the other hand, patients who were on ART regimen groups d4t-3TC-NVP and d4t-3TC-EFV at baseline and those who experienced regimen change from their baseline for reasons other than ADR were at higher risk of developing ADRs (Table 4).

Table 4: Cox regression analysis of the relationship between explanatory variables and the time to ADR development, Debre Markos Referral Hospital, 2008 - 2018. 
Survival Status

$\begin{array}{lcccc}\text { Variables } & \text { Event (ARD) } & \text { Censored } & \text { CHR }(95 \% \text { C.I }) & \text { AHR }(95 \% \text { C.I }) \\ \text { Gender } & & & & \\ \text { Male } & 18 & 177 & 1.00 & 1.00 \\ \text { Female } & 49 & 241 & 2.01(1.17,3.46) & 1.62(0.91,2.89)\end{array}$

\section{Occupation}

\begin{tabular}{|c|c|c|c|c|}
\hline Contract Employee & 33 & 272 & 1.00 & 1.00 \\
\hline Sex workers and drivers & 10 & 27 & $2.14(1.06,4.35)$ & $2.78(1.31,5.92) * \star$ \\
\hline Merchant & 5 & 4 & $5.77(2.24,14.81)$ & $2.63(0.81,8.48)$ \\
\hline Farmer & 11 & 41 & $2.29(1.16,4.53)$ & $2.51(1.22,5.16)$ * \\
\hline Government Employee & 8 & 74 & $0.75(0.34,1.62)$ & $1.19(0.53,2.71)$ \\
\hline \multicolumn{5}{|l|}{ Residence } \\
\hline Within catchment area & 28 & 245 & 1.00 & 1.00 \\
\hline Out of catchment area & 39 & 173 & $1.72(1.06,2.79)$ & $1.73(1.04,2.86)$ * \\
\hline \multicolumn{5}{|l|}{ Baseline WHO stage } \\
\hline Stage I and II & 25 & 245 & 1.00 & 1.00 \\
\hline Stage III and IV & 42 & 173 & $2.81(1.71,4.62)$ & $2.59(1.54,4.36) * \star \star$ \\
\hline \multicolumn{5}{|l|}{ Baseline regimen } \\
\hline $1 c+1 d+1 e+1 f$ & 11 & 322 & 1.00 & 1.00 \\
\hline $1 a+1 b$ & 54 & 90 & $7.99(4.18,15.30)$ & $4.03(1.98,8.20)$ *** \\
\hline $1 g+1 h$ & 2 & 6 & $6.59(1.46,29.72)$ & $4.57(0.79,26.34)$ \\
\hline
\end{tabular}

Baseline CD4

$<=200$

52

239

1.00

$>200$

15

179

$0.62(0.35,1.09)$

$1.10(0.60,2.05)$

Regimen changed

$\begin{array}{lllll}\text { Yes } & 62 & 125 & 16.63(6.68,41.37) & 9.99(3.79,26.28) * \star \star \\ \text { No } & 5 & 293 & 1.00 & 1.00\end{array}$

Ever took CPT

Yes

49

323

1.00

1.00 
No

Anti TB prophylaxis

\begin{tabular}{lllll}
\hline Yes & 43 & 286 & $1.32(0.80,2.18)$ & $2.83(1.61,4.96) \star \star \star$ \\
\hline No & 24 & 132 & 1.00 & 1.00
\end{tabular}

Functional status

\begin{tabular}{lllll}
\hline Working & 43 & 319 & 1.00 & 1.00 \\
\hline Ambulatory & 20 & 86 & $1.35(0.79,2.3)$ & $1.43(0.77,2.64)$ \\
\hline Bed ridden & 4 & 13 & $1.61(0.58,4.48)$ & $1.38(0.43,4.45)$ \\
\hline
\end{tabular}

$1 \mathrm{a}=\mathrm{d} 4 \mathrm{t}-3 \mathrm{TC}-\mathrm{NVP}, 1 \mathrm{~b}=\mathrm{d} 4 \mathrm{t}-3 \mathrm{TC}-\mathrm{EFV}, 1 \mathrm{c}=$ AZT-3TC-NVP, 1d= AZT-3TC-EFV, 1e= TDF-3TC-EFV, $1 \mathrm{f}=\mathrm{TDF}+3 \mathrm{TC}+\mathrm{NVP}, 1 \mathrm{~g}=\mathrm{ABC}+3 \mathrm{TC}+\mathrm{EFV}, 1 \mathrm{~h}=\mathrm{ABC}+3 \mathrm{TC}+\mathrm{NVP}, \mathrm{CPT}=$ cotrimoxazole preventive therapy, *** significant at $p$ value of $0.001, * *$ significant at $p$ value of $0.01, *$ significant at $p$ value of 0.05

\section{Discussions}

Antiretroviral toxicity is an increasingly important issue in the management of HIV-infected patients. Early start of ART, opportunistic complications decrements and the chronic nature of HIV resulted in more drugs being used in more patients for longer periods, which can increase the likelihood of ADRs development [15].

In this study, we estimated the incidence and identified important predictors of ADR development. The incidence rate of adverse drug reaction was 3 (95\% C.I: $2.4,3.86)$ per 100-person years of follow up. This is lower than findings from Bahir Dar city (4.3/100 PY) [16], finding from seven teaching hospitals in Ethiopia (9/100 PY) [17], two retrospective studies from Nigeria (4.6/100 PY and 4.05/100 PY) [18, 19] and another study in Ethiopia [11]. This lower figure might be related to the difference in follow up duration in which all of these previous studies had shorter duration than the current study. As the follow up time increased the denominator will be larger thereby incidence rate becomes smaller. The incidence was higher among females than males 3.85/100 PY and 1.93/100 PY respectively. This finding is in line with a finding of a follow study form Nigeria [19]. This might be due to the fact that women are more vulnerable for regimen changes than men for example during pregnancy.

About $13.81 \%$ (95\% C.I: $10.87 \%, 17.2 \%)$ of the cohort had experienced ADR. This figure is higher than findings from Bahir Dar city (10\%) [16], a tertiary hospital in Addis Ababa (7.7\%) [20], seven teaching hospitals in Ethiopia (9.5\%) [17], Nigeria (1.4\%) [18, 19], a tertiary hospital in Ghana (9.4\%) [21] and India (2.4\%) [22]. The difference might be due to the longer follow up time in the current study which can be explained by the longer the follow up the higher the probability of including the ADR incidences. The main reason for ADR development in several literatures are also switching ART medicines for different reasons and taking concomitant medications including cotrimoxazole and Isoniazid preventive therapies, in which all of these are at higher proportions in this study $(38.6 \%, 32.2 \%$ and $23.3 \%)$ respectively. 
Half of the cohort (52.37\%) experienced regimen changes, of which $26.37 \%$ were due to adverse drug reactions. This finding is lower than findings from Gondar referral Hospital where side effects were the commonest (70.45\%) [23] reasons for regimen change, Jimma Southwest Ethiopia where the change due to ADRs accounted for $48.94 \%$ of all changes [24] and Kenya where it accounted for $66.3 \%$ [25]. This can be explained by the proportion of $d 4 T$ which causes majority of regimen changes is lower $(29.7 \%)$ in this study than the previous studies in Jimma Southwest Ethiopia and Kenya. In the other hand, it is higher than a finding from Nigeria where the overall prevalence of regimen change was $73.3 \%$ and ADRs attributed to $10 \%$ [26] which can be explained by the difference in follow up duration.

Patients with baseline regimen containing stavudine in the combination were at higher risk of developing adverse drug reactions. This is in agreement with previous literatures [18, 24, 27], and it might be because stavudine is known for its frequent side effects on the abdominal fat distribution and other several organs [28].

Patients with baseline WHO clinical stage of III and IV were 2.56 times at higher risk to develop adverse drug reactions compared to WHO stages I and II. This finding is in line with findings in Bahir Dar city Northwest Ethiopia [16], Hiwot Fana specialized Hospital Eastern Ethiopia [27], Jos University Teaching Hospital in Nigeria [26], and a study from India [7]. This might be due to the fact that patients in the advanced clinical stage of HIV are more vulnerable for multiple drug intake for the treatments of opportunistic infections. In the other hand, patients on advanced clinical stages are less resistant to drug side effects and are more likely to change regimens, thereby will find themselves at higher risk of drug reactions [29].

Patients who had ever taken anti-TB medicines were found at higher risk of severe adverse drug reactions development than their counter parts. This is in line with literatures revealing HIV-infected patients receiving TB treatment commonly experience drug toxicity [30,31], even some findings recommended deferring of ARVs during the intensive phase of TB treatment [31, 32].

Coming from outside of the catchment areas was also another predictor factor for developing adverse drug reactions. It might have an implication that distance can affect the consistency of drug refiling and might affect getting close follow up and advises from service providers. In the other hand, it can be also related to the lack of social support as patients frequently go far out of their nearby facility due to fear of stigma and discriminations.

The hazard of ADR development among those who had experience of regimen change for reasons other than ADRs was higher compared to those who stayed on a single regimen during their follow up period. This can be due to the fact that being exposed for multiple ARV drugs even sequentially can increase the risk of drug adverse reactions. It has also an implication of either drug stock out, treatment failure or new TB infection, which are the main reasons for regimen change other than ADRs could contribute for the likelihood of ADR development. 
Occupational status of patients also showed statistically significant association with ADRs, where commercial sex workers and car drivers were at higher risk for ADRs compared to those with contract works. This can be due to the fact that their work nature affects appropriate drug taking and they are vulnerable for multiple sexual partners which can increase the likelihood of ADR development. They are also more exposed for alcohol intake and it can cause ADRs. Farmers were also at higher risk than contract workers. This also might have related to their work nature, health facility accessibility and level of awareness, as farmers come out of Debre Markos town.

\section{Limitation of the study}

Since this study used secondary data, it has limitation of including all possible explanatory variables exhaustively. We could not also describe and discuss the detailed profiles of each specific adverse drug reaction.

\section{Conclusion}

The incidence of adverse drug reactions was relatively lower than reported in different parts of Ethiopia and other African countries. However, the overall burden in the 10 years period was still high. Base line regimen type, regimen change for different reasons, taking anti tuberculosis prophylaxis or anti tuberculosis treatments, residing outside of the catchment area, and having advanced WHO clinical stages at baseline and working as commercial sex worker and car driver were found as independent predictors of adverse drug reactions in this retrospective follow up study.

Health professionals working in the ART clinic need to give special attention for commercial sex workers and drivers and patients on advanced WHO clinical stages to prevent ADR development among these groups. Strict follow up of patients taking TB concomitant drugs is important. Health professionals also should consult patients coming from outside of the catchment area to attend their follow up in the nearby ART center. Further prospective follow up study needs to be conducted to incorporate all possible contributing factors and to have detailed specific ADR profiles.

\section{Abbreviations}




\begin{tabular}{ll} 
3TC & Lamivudine \\
\hline ABC & Abacavir \\
\hline AIDS & Acquire Immune-deficiency Syndrome \\
\hline ART & Antiretroviral Therapy \\
\hline ARV & Antiretroviral (drugs) \\
\hline AZT/ZDV & Zidovudine \\
\hline BMI & Body Mass Index \\
\hline CD4 & Type of T-lymphocyte, white blood cells \\
\hline CPT & Cotrimoxazole Preventive Therapy \\
\hline d4T & Stavudine \\
\hline HAART & Highly active antiretroviral therapy \\
\hline HIV & Human immunodeficiency Virus \\
\hline HR & Hazard Ratio \\
\hline IPT & Isoniazid Preventive Therapy \\
\hline LMM & Linear Mixed Model \\
\hline PLHIV & People living with HIV \\
\hline PY & Person Year \\
\hline TB & Tuberculosis \\
\hline TDF & Tenofovir \\
\hline WHO & World Health Organization \\
\hline
\end{tabular}

\section{Declarations}

\section{Ethics approval and consent to participate}

Ethical approval was obtained from the ethical review committee of University of Gondar College of Medicine and health sciences. Letter of cooperation was sought for Debre Markos referral Hospital. Furthermore, confidentiality was maintained for all forms of information using anonymous medical registration numbers as identification.

\section{Consent for publication}

Not applicable 
Availability of data and material

All the data supporting the study findings are within the manuscript. Additional detailed information and raw data will be shared upon request addressed to the corresponding author.

Competing interests

The authors declare that they have no competing interests.

\section{Funding}

No funding has been obtained from any source to carry out this study.

Authors' contributions

GDK wrote the proposal, did the analysis and prepared the manuscript.

TAA and AT revised the proposal and the manuscript. All the authors have read and approved the manuscript.

\section{Acknowledgements}

We would like to thank data collectors and supervisors for their commitment throughout the data retrieval period.

\section{References}

1. HIV/AIDS JUNPo: Fact sheet-Latest statistics on the status of the AIDS epidemic. In.; 2017.

2. UNAIDS, WHO: Global AIDS monitoring. In. Geneva: UNAIDS; 2017.

3. UNAIDS: State of the AIDS epidemic. In.: Joint United Nations Programme on HIV/AIDS (UNAIDS); 2017.

4. HIV/AIDS JUNPo: Global AIDS update 2016. Geneva: UNAIDS 2016.

5. Edwards IR, Aronson JK: Adverse drug reactions: definitions, diagnosis, and management. The lancet 2000, 356(9237):1255-1259.

6. WHO: Consolidated guidelines on the use of antiretroviral drugs for treating and preventing HIV infection: recommendations for a public health approach: World Health Organization; 2016.

7. Shet A, Antony J, Arumugam K, Kumar Dodderi S, Rodrigues R, DeCosta A: Influence of adverse drug reactions on treatment success: prospective cohort analysis of HIV-infected individuals initiating first-line antiretroviral therapy in India. PloS one 2014, 9(3):e91028. 
8. HIV/AIDS JUNPo, HIV/Aids JUNPo: 90-90-90: An ambitious treatment target to help end the AIDS epidemic. Geneva: UNAIDS 2014.

9. WHO: Surveillance of antiretroviral toxicity: technical review meeting on country experiences in antiretroviral toxicity surveillance: sharing preliminary results and lessons learnt, identifying solutions, 7-8 November 2013, Geneva, Switzerland: meeting report. 2014.

10. WHO: Surveillance of antiretroviral drug toxicity within antiretroviral treatment programmes: technical brief. 2013.

11. Bezabhe WM, Bereznicki LR, Chalmers L, Gee P, Kassie DM, Bimirew MA, Peterson GM: Adverse drug reactions and clinical outcomes in patients initiated on antiretroviral therapy: a prospective cohort study from Ethiopia. Drug safety 2015, 38(7):629-639.

12. HAPCO: Country/Regional Operational Plan. April 21, 2017.

13. Royston P, Parmar MK: Restricted mean survival time: an alternative to the hazard ratio for the design and analysis of randomized trials with a time-to-event outcome. BMC medical research methodology 2013, 13(1):152.

14. Bruin J: newtest: command to compute new test. UCLA: Statistical Consulting Group. Retrieved 2006, 6:2016.

15. Carr A, Cooper DA: Adverse effects of antiretroviral therapy. The Lancet 2000, 356(9239):1423-1430.

16. Kindie E, Alamrew Anteneh Z, Worku E: Time to development of adverse drug reactions and associated factors among adult HIV positive patients on antiretroviral treatment in Bahir Dar City, Northwest Ethiopia. PloS one 2017, 12(12):e0189322.

17. Gudina EK, Teklu AM, Berhan A, Gebreegziabhier A, Seyoum T, Nega A, Medhin G, Kebede A, Assefa Y: Magnitude of Antiretroviral Drug Toxicity in Adult HIV Patients in Ethiopia: A cohort study at seven teaching hospitals. Ethiopian journal of health sciences 2017, 27(Suppl 1):39-52.

18. Abah IO, Akanbi M, Abah ME, Finangwai Al, Dady CW, Falang KD, Ebonyi AO, Okopi JA, Agbaji OO, Sagay AS et al: Incidence and predictors of adverse drug events in an African cohort of HIV-infected adults treated with efavirenz. Germs 2015, 5(3):83-91.

19. Eluwa GI, Badru T, Akpoigbe KJ: Adverse drug reactions to antiretroviral therapy (ARVs): incidence, type and risk factors in Nigeria. BMC clinical pharmacology 2012, 12(1):7.

20. Abdissa SG, Fekade D, Feleke Y, Seboxa T, Diro E: Adverse drug reactions associated with antiretroviral treatment among adult Ethiopian patients in a tertiary hospital. Ethiopian medical journal 2012, 50(2):107-113. 
21. Lartey M, Asante-Quashie A, Essel A, Kenu E, Ganu V, Neequaye A: Adverse drug reactions to antiretroviral therapy during the early art period at a tertiary hospital in Ghana. The Pan African medical journal 2014, 18.

22. Anwikar SR, Bandekar MS, Smrati B, Pazare AP, Tatke PA, Kshirsagar NA: HAART induced adverse drug reactions: a retrospective analysis at a tertiary referral health care center in India. The International journal of risk \& safety in medicine 2011, 23(3):163-169.

23. Anlay DZ, Alemayehu ZA, Dachew BA: Rate of initial highly active anti-retroviral therapy regimen change and its predictors among adult HIV patients at University of Gondar Referral Hospital, Northwest Ethiopia: a retrospective follow up study. AIDS research and therapy 2016, 13:10.

24. Birlie B, Braekers R, Awoke T, Kasim A, Shkedy Z: Multi-state models for the analysis of time-totreatment modification among HIV patients under highly active antiretroviral therapy in Southwest Ethiopia. BMC infectious diseases 2017, 17(1):453.

25. Inzaule S, Otieno J, Kalyango J, Nafisa L, Kabugo C, Nalusiba J, Kwaro D, Zeh C, Karamagi C: Incidence and predictors of first line antiretroviral regimen modification in western Kenya. PloS one 2014, 9(4):e93106.

26. Abah IO, Darin KM, Ebonyi AO, Ugoagwu P, Ojeh VB, Nasir N, Falang KD, Olaitan O, Agbaji O, Idoko J et al: Patterns and Predictors of First-Line Antiretroviral Therapy Modification in HIV-1-Infected Adults in a Large Urban Outpatient Cohort in Nigeria. Journal of the International Association of Providers of AIDS Care 2015, 14(4):348-354.

27. Weldegebreal F, Mitiku H, Teklemariam Z: Magnitude of adverse drug reaction and associated factors among HIV-infected adults on antiretroviral therapy in Hiwot Fana specialized university hospital, eastern Ethiopia. Pan African Medical Journal 2016, 24(1).

28. Roca B, Gómez CJ, Arnedo A: Stavudine, lamivudine and indinavir in drug abusing and non-drug abusing HIV-infected patients: adherence, side effects and efficacy. Journal of Infection 1999, 39(2):141145.

29. Berheto TM, Haile DB, Mohammed S: Predictors of loss to follow-up in patients living with HIV/AIDS after initiation of antiretroviral therapy. North American journal of medical sciences 2014, 6(9):453.

30. Patel A, Patel K, Patel J, Shah N, Patel B, Rani S: Safety and antiretroviral effectiveness of concomitant use of rifampicin and efavirenz for antiretroviral-naive patients in India who are coinfected with tuberculosis and HIV-1. JAIDS Journal of Acquired Immune Deficiency Syndromes 2004, 37(1):11661169 .

31. Dean GL, Edwards SG, Ives NJ, Matthews G, Fox EF, Navaratne L, Fisher M, Taylor GP, Miller R, Taylor $\mathrm{CB}$ : Treatment of tuberculosis in HIV-infected persons in the era of highly active antiretroviral therapy. 
Aids 2002, 16(1):75-83.

32. Yee D, Valiquette C, Pelletier M, Parisien I, Rocher I, Menzies D: Incidence of serious side effects from first-line antituberculosis drugs among patients treated for active tuberculosis. American journal of respiratory and critical care medicine 2003, 167(11):1472-1477.

\section{Figures}

\section{Overall survival history of the cohort}

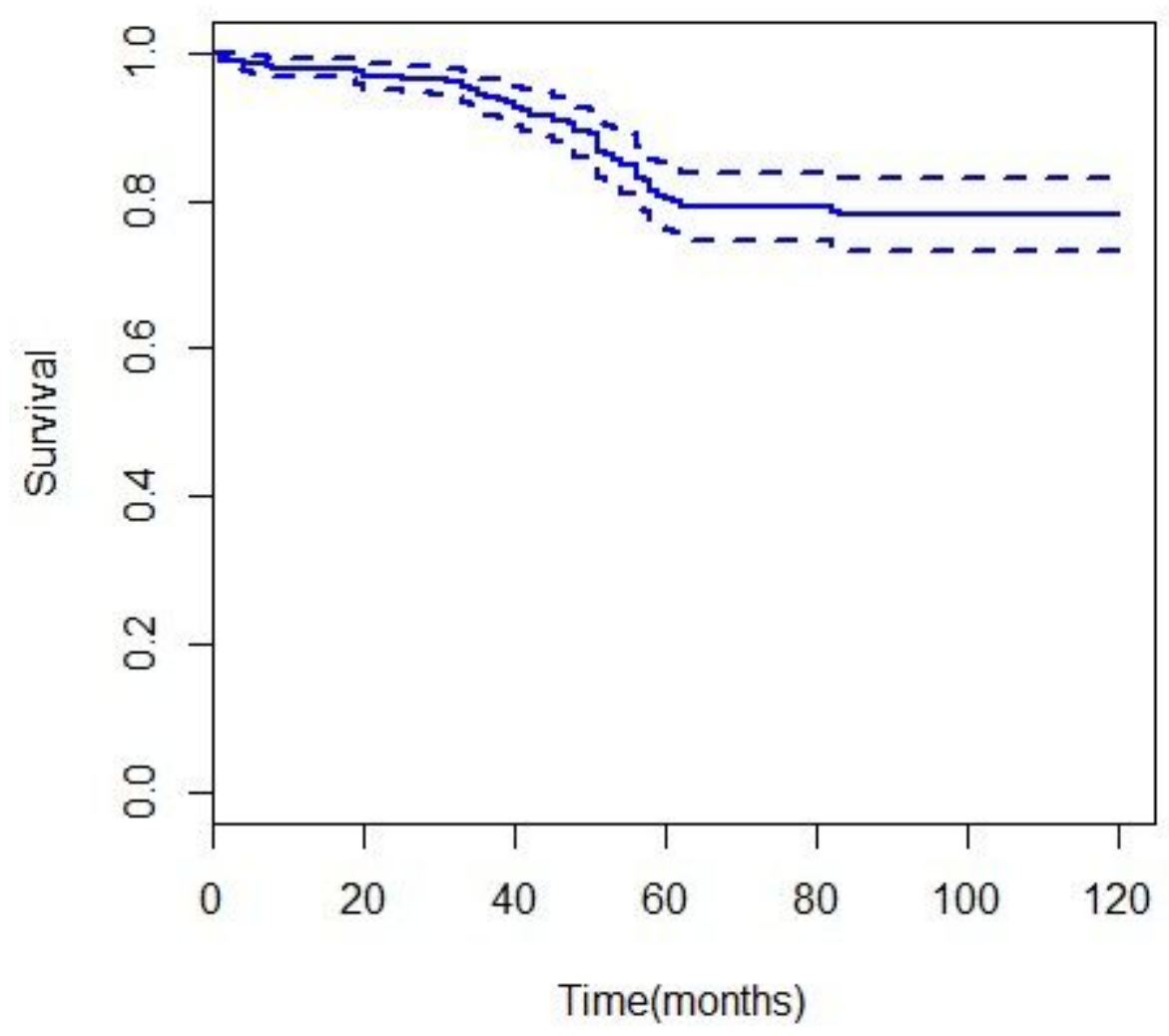

Figure 1

Cumulative survival Kaplan Meier curve for time to the development of ADRs among HIV patients on ART, Debre Markos Referral Hospital. 


\section{KM survival curve by gender}

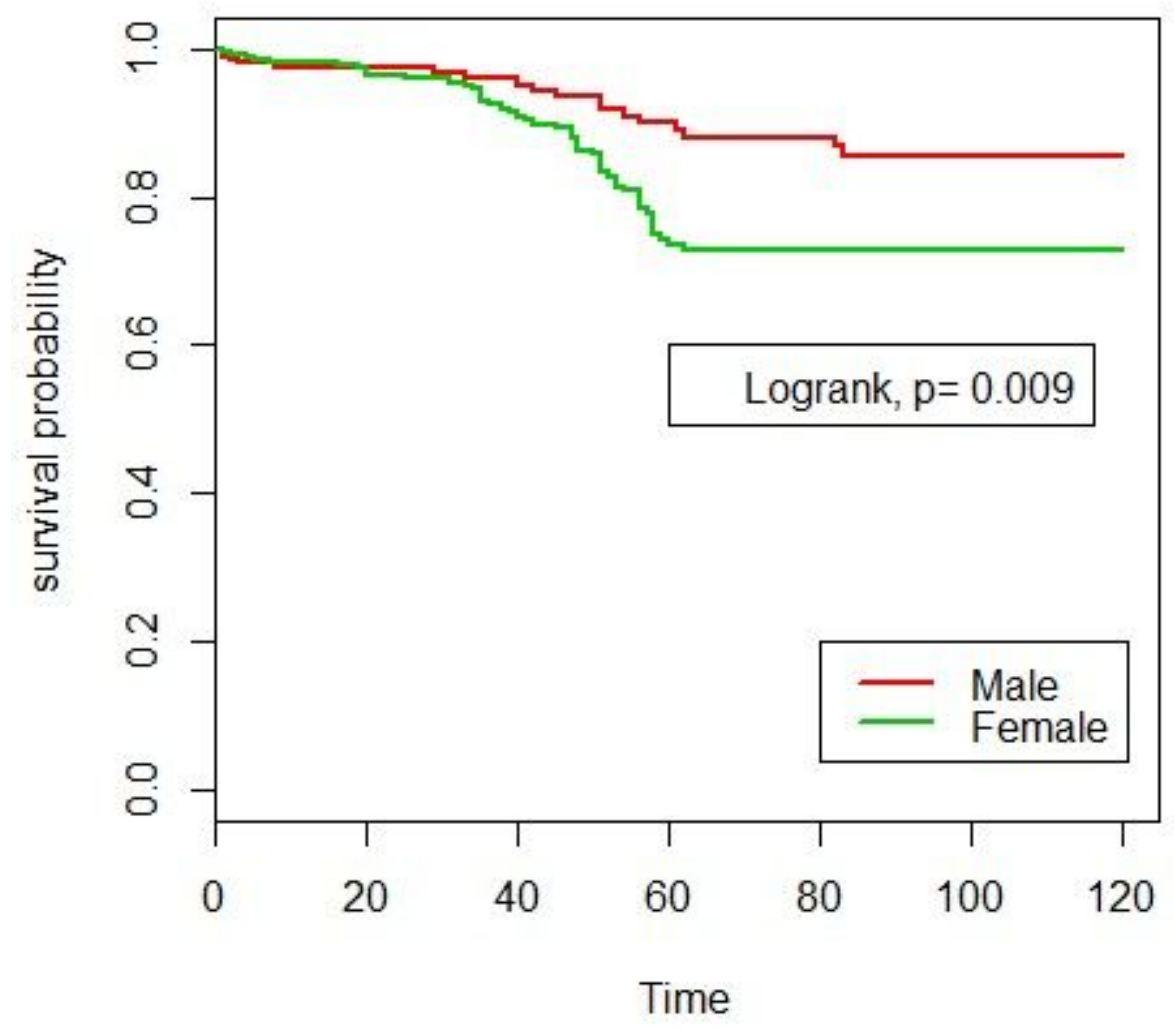

\section{Figure 2}

Kaplan Meier curves for time to the development of ADRs among HIV patients on ART by gender, Debre Markos Referral Hospital 2008 to 2018. 


\section{KM survival curve by residence}

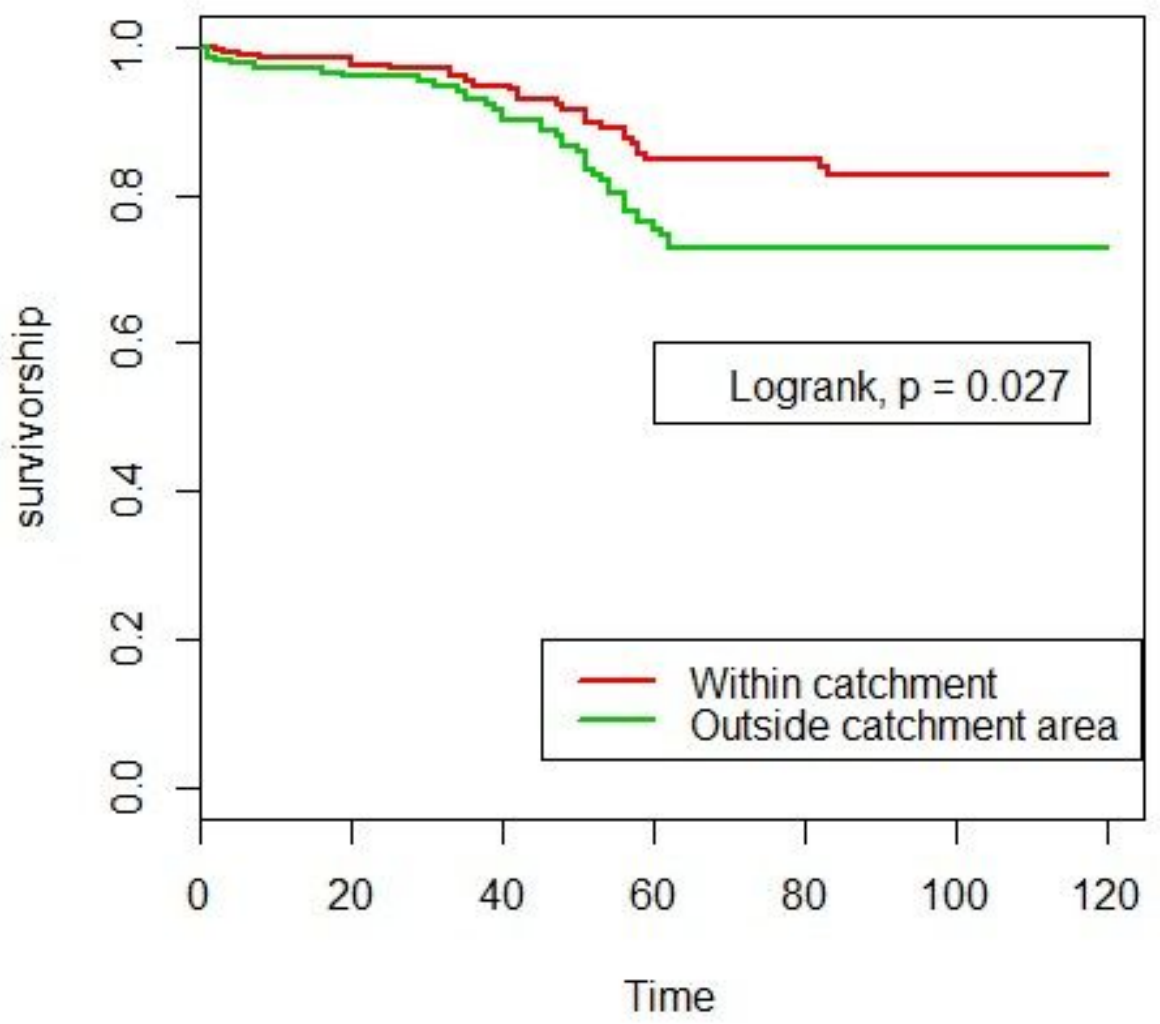

Figure 3

Kaplan Meier curves for time to the development of ADRs among HIV patients on ART by source of residence, Debre Markos Referral Hospital 2008 to 2018. 


\section{Goodness of fit test for Cox PH model}

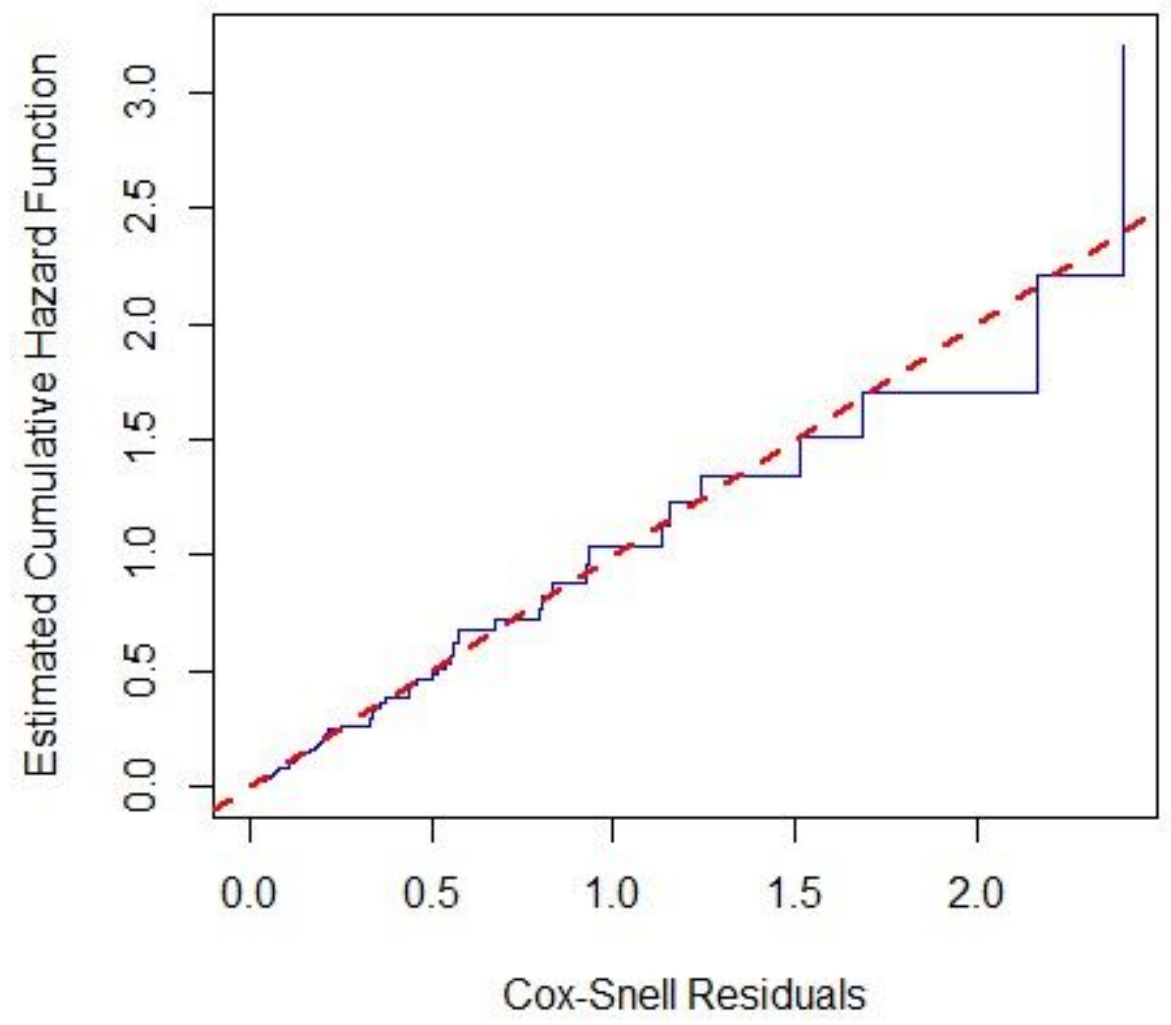

Figure 4

Goodness of fit of the final Cox proportional hazards regression model using Cox Snell Residuals. 